\title{
RESULTADOS VS. REALIDAD: UN EJEMPLO EN LAS ACTITUDES LINGÜÍSTICAS
}

\author{
RESULTS VS. REALITY: AN EXAMPLE IN LINGUISTIC ATTITUDES
}

\section{Raquel Ruiz Pareja}

Universidad de Tromsø

Resumen

Este artículo presenta una revisión de parte de un estudio de investigación sobre actitudes lingüísticas: "Estudio sobre las actitudes lingüísticas en Andalucía: andaluz oriental y andaluz occidental" (Ruiz Pareja, 2015). El objetivo de este trabajo es arrojar un poco de luz sobre el contraste que existe en Sociolingüística entre las actitudes afectivas y cognitivas, que además están presentes en numerosos cuestionarios y estudios en esta disciplina. Para ello se repite parte del cuestionario original, donde parecía haber cierta contradicción entre actitudes y comportamientos. Los nuevos resultados indican que esta contradicción sigue existiendo; no obstante, ésta parece explicarse con la teoría del conocimiento implícito y explícito en Psicología y algunas hipótesis derivadas de ella.

Palabras clave: Actitudes lingüísticas,
conocimiento implícito,
explícito, sociolingüística

Abstract

This article presents a review of part of a research study about linguistic attitudes: "Study about linguistic attitudes in Andalusia: Eastern Andalusia and Western Andalusia" (Ruiz Pareja, 2015). The aim of this paper is to shed light on the contrast that exists in Sociolinguistics between affective and cognitive attitudes, which are also present in many questionnaires and studies in this discipline. In order to achieve it, part of the original questionnaire, where it seemed to exist a contradiction between attitudes and behaviors, will be repeated. The new results indicate that this contradiction continues to exist; however, this may be explained by the implicit and explicit theory of knowledge in Psychology, and some hypotheses derived from it.

KEY WORDS: Linguistic attitudes, implicit knowledge, explicit knowledge, 


\section{INTRODUCCIÓN}

En este artículo comenzaremos tratando el tema del español de Andalucía, más específicamente el de Cádiz, desde la perspectiva de las distintas actitudes lingüísticas, analizando las percepciones que tienen los hablantes de su propia variedad en comparación con otras que les pueden ser más o menos afines.

El modo de recopilación de datos que usaremos será un cuestionario, cuyos resultados compararemos con otro cuestionario similar del año 2015. Precisamente, el uso de este mismo método, que al principio puede parecer fiable, es lo que nos llevará a la cuestión principal de este trabajo: ¿reflejan los resultados de este tipo correctamente la realidad? Si no fuera así, ¿por qué ocurre esto? ¿Habría que descartarlos, o bien existen otras maneras de interpretarlos?

Para responder a estas cuestiones usaremos distintas teorías e hipótesis en Psicología; no ahondaremos demasiado en su teoría, pero sí que las intentaremos comparar con nuestros resultados y otros casos prácticos, y nos servirán para encontrar posibles explicaciones.

Al final recopilaremos nuestras conclusiones y veremos cuáles son las posibles razones por las que a veces los resultados de distintos cuestionarios no corresponden con la realidad, y cómo se podrían interpretar cuando esto ocurre.

\section{TRASFONDO TEÓRICO}

El tema de las actitudes suscita mucho interés no sólo en Psicología (donde se origina), sino también en muchas otras disciplinas: en nuestro estudio, vamos a estar trabajando todo el tiempo sobre lo que en Sociolingüística se denomina "actitudes lingüísticas". La Sociolingüística usa la teoría de las actitudes para ver cómo el hablante percibe su lengua, su variedad (o incluso lenguas o variedades que le sean ajenas) y qué relación se establece entre ellos.

Ruiz Pareja define las actitudes como "opiniones, ideas o creencias, que se refieren, por una parte, a la percepción que tiene el individuo sobre la lengua en sí, y por otra, a la relación entre lengua y sociedad". Dentro de estas actitudes, se distinguen aquellas basadas en el conocimiento o las impresiones que tenemos sobre algo (actitudes cognitivas), y las que implican un sentimiento sobre ese algo (actitudes afectivas). (Ruiz Pareja, 2015: 9-10). Esto es la base para comprender tanto el objetivo del estudio anterior como el nuestro de ahora.

Ruiz Pareja dedica su estudio a analizar las diferencias de percepción que tienen los propios hablantes sobre estas dos variedades, tomando como base la distinción cognitividad-afectividad planteada en Trafimow y Sheeran (Trafimow, 2005). El método que se utiliza es un cuestionario al que contestan dos grupos de hablantes bien definidos: 18 personas de Cádiz (capital y provincia) y 4 de Almería, éste último como grupo de control. 
Las preguntas están divididas según en qué actitudes estén enfocadas (Ruiz Pareja, 2015: 16).

De todas las preguntas del cuestionario, nos centraremos en la n. 8 del cuestionario de Cádiz, que es la que nos interesa por haber cierta incongruencia por parte de Ruiz Pareja en cuanto a los resultados que se obtuvieron. Las preguntas son las siguientes:

8.a “¿Se podrían cantar chirigotas con acento/dialecto de Almería?”
8.b “¿Se podrían cantar chirigotas con acento/dialecto de Madrid?”.

De los 18 participantes, sólo uno respondió negativamente a ambas preguntas. En cuanto a esto, se afirma lo siguiente: «(...) estoy segura, por experiencia propia, de que la gran mayoría de los encuestados verían una chirigota de Ciudad Real, por ejemplo, y no se encontrarían cómodos y les sería más difícil reírse tanto como con una de Cádiz» (Ruiz Pareja, 2015: 40). Tras esto, intenta ahondar un poco en el tema, pero se queda muy en la superficie al no dar razones de por qué; simplemente se limita a describir la relación dialecto-folclore, lo que, precisamente, daría resultados contrarios.

Puesto que ese interrogante quedaba sin respuesta, se ha repetido ahora parte del cuestionario, esta vez con un mayor número de participantes, con la intención de obtener (en principio) datos que fueran un tanto más fiables, y ver si esta tendencia se repetía, o bien fue algo puntual.

\section{EXPOSICIÓN DE LOS RESULTADOS}

El grupo de encuestados consta ahora de 30 personas (15 hombres y 15 mujeres), comprendidas entre los 20 y 40 años. Las preguntas se distribuyeron vía Google Forms a amigos y conocidos con diferentes perfiles. La razón era que el cuestionario pudiera llegar a más personas que el original, que fue repartido en papel a amigos, familiares y conocidos. También fue la razón de que se le añadiera la aclaración "Entendiendo se podrían como adecuado, o que funciona", y se les pedía justificar sus respuestas: con ello se pretendía contrarrestar el elemento no presencial del entrevistador. Como veremos más adelante, quizás estas aclaraciones no fueran del todo acertadas. Presentamos entonces aquí los nuevos resultados, comparados con los anteriores: 

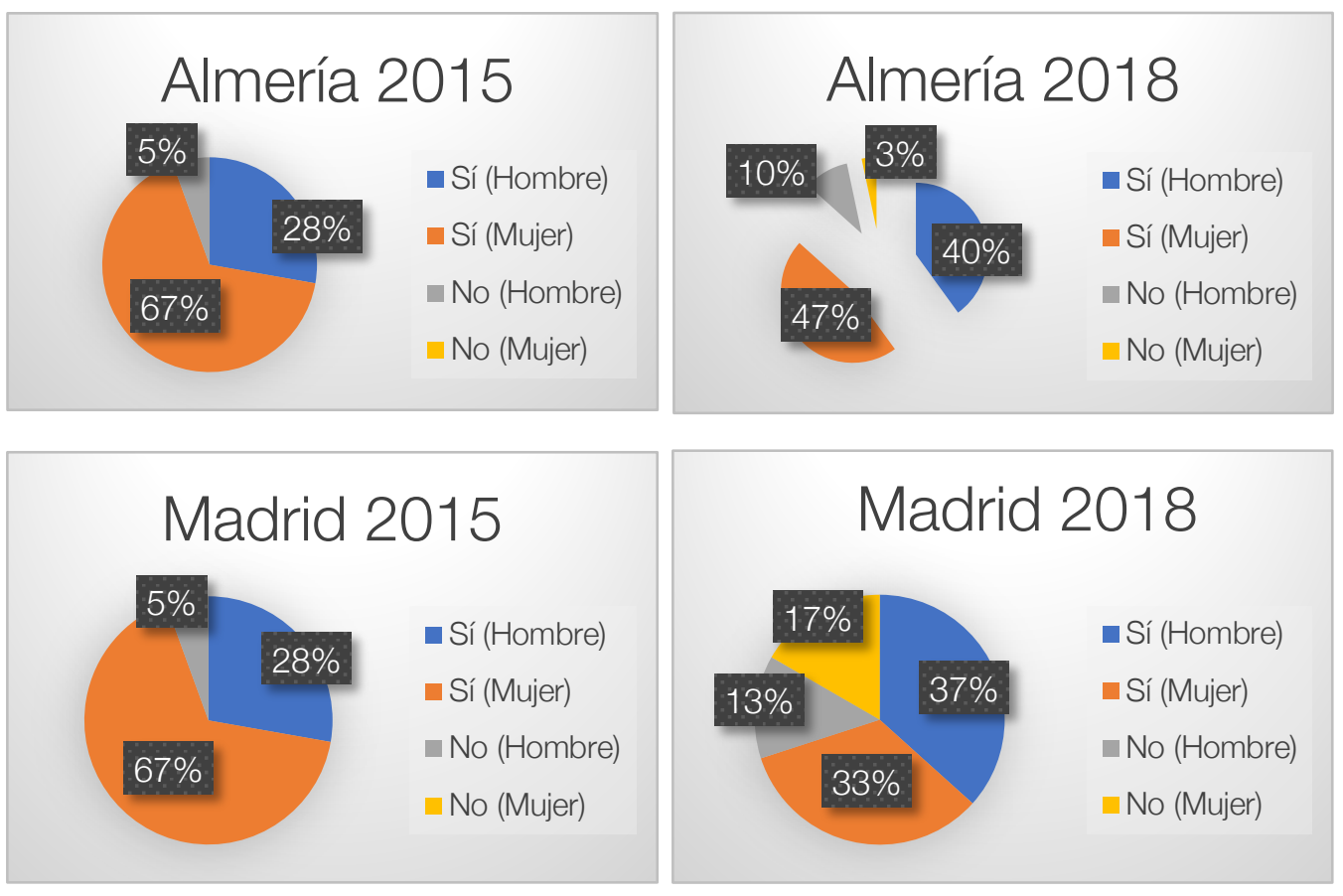

Comparando estos resultados con los originales (Ruiz Pareja, 2015: 39-40), vemos que igualmente el porcentaje de respuestas positivas es alto. En el caso del dialecto de Madrid es donde quizás haya un poco más de diferencia: en el cuestionario original sólo 1/18 respondía negativamente, mientras que aquí tenemos 9/30 (en porcentajes, 30\% frente a $5 \%)$. En nuestra versión, mientras que la mayor parte de hombres y casi la totalidad de mujeres apoyan la idea de la chirigota de Almería, en la de Madrid se encuentra un poco más repartida: un tercio de las mujeres y casi un tercio de los hombres respondieron negativamente.

Debido a la forma en la que se recopilaron los resultados, no pudo haber control de cómo respondían los participantes, y, aunque la pregunta incluía justificar la respuesta, no todos lo hicieron: cinco personas dejaron su respuesta sin justificación. En las otras 25 respuestas, se encuentran comentarios muy interesantes, algunos muy similares entre sí. Sobre las chirigotas con acento/dialecto de Madrid tenemos, por ejemplo, los siguientes:

\footnotetext{
- Podría ser igualmente posible, aunque perdería la posibilidad de tener la identificación del pueblo gaditano y andaluz frente al acento y la idiosincrasia humorística de la región. (Hombre, 28 años). - Sí, creo que todo el mundo tiene derecho a crear su propia chirigota, la diferencia de acento puede darle originalidad, aunque perdería en gracia (Hombre, 31 años).
}

Tenemos, por otra parte, a más participantes que dan una respuesta positiva a las chirigotas con acento/dialecto almeriense. Esto puede deberse, bien al desconocimiento de los rasgos de dicho dialecto (podría ser que se percibiera como similar al de Cádiz), o bien al hecho de contraponerlo con dialecto madrileño, pues este se considera una variedad muy estandarizada, y por tanto lejana a cualquier dialecto de Andalucía. Con esto tienen que ver las diferentes jerarquías dialectales (Ruiz Pareja, 2015: 12), donde, en este 
caso, el español de Almería y el español de Cádiz formarían un bloque (español andaluz) opuesto al español de Madrid, no sólo en cuanto a geografía y similitudes dialectales, sino a estatus.

Entre los comentarios de las preguntas sobre dialecto de Almería destacamos los siguientes:

\begin{abstract}
- Si, quizás sonaría raro y no se entendería bien, pero es posible. Al igual que hay chirigotas de Sevilla y tienen éxito frente al público gaditano (Hombre, 28 años). Aquí vemos ese posible desconocimiento de los rasgos dialectales al comparar Sevilla y Almería. Ambas variedades están desconectadas dentro del continuum dialectal español, lo que significa que existe más de un rasgo que las distingue entre sí (Coloma, 2013: 375). Ningún hablante que fuera consciente de estas diferencias concebiría el dialecto almeriense similar al de Sevilla.

- No, porque tienen un acento muy cerrado y es más complicado de entender (Mujer, 33 años). Aquí los términos "cerrado" y "complicado de entender" son ambiguos y muy relativos, así que no es posible afirmar con seguridad que esto se deba al desconocimiento del dialecto de Almería.
\end{abstract}

Para hacer un análisis un poco más exhaustivo de los resultados, utilizaremos aquí las justificaciones y comentarios para ver con qué tipo de hablantes nos encontramos, según la adaptación que hace Haddock de Chaiken et al. sobre la correlación actitud-cognitividad y actitud-afectividad. Grosso modo, su teoría se resume en que distintos individuos se clasificarían en distintos grupos, según estén relacionadas entre sí sus actitudes cognitivas y afectivas. En el siguiente cuadro vemos más detalladamente de qué se trata (Haddock y Maio, 2004: 40).

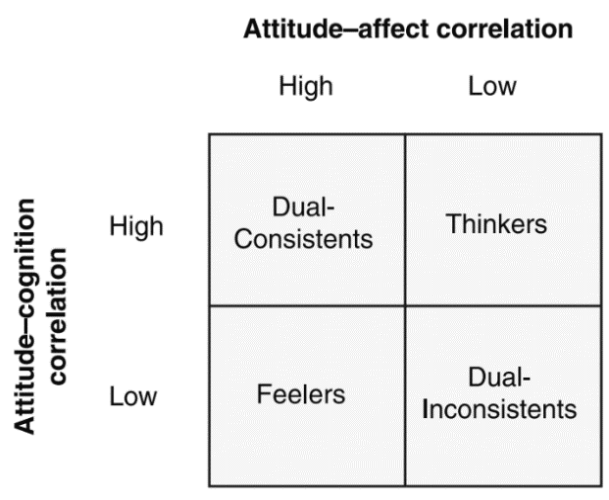

Haddock llama dual-consistents a aquellos individuos que presentan tanto una cognitividad como una afectividad fuertes. En este grupo incluiríamos a aquellos participantes que dieron respuestas del tipo "Sí se podría, aunque no sonaría igual" (sí se podría porque, por ejemplo, hubiera unas reglas para hacer chirigota, pero, sin embargo, no sonaría igual porque si el dialecto se cambia, la relación afectiva no es la misma o se pierde). Si vemos los resultados para Almería de aquellos que sí que justificaron su respuesta, tendríamos a 4, y para Madrid tendríamos 5.

Tenemos por otro lado a los thinkers: aquellos individuos que dan respuestas donde predomina la cognitividad sobre la afectividad. Aquí pondríamos las respuestas del tipo "Sí 
se podría, no importan los acentos". En este grupo tenemos 18 para Almería, y 14 para Madrid.

Los feelers, por otro lado, son lo contrario a los thinkers: sus respuestas están cargadas de afectividad, pero de poca cognitividad. Por ejemplo, en respuestas tipo "No se podría, porque no tendría la misma gracia". Para Almería encontramos 2, y para Madrid 5.

Por último, tenemos lo que Haddock denomina como dual inconsistents, que serían aquellos individuos en los que encontramos tanto baja afectividad como baja cognitividad. Podrían ser respuestas tipo "Me da igual", "No me importa", o quizás simplemente "No sé". De éstas no tenemos ninguna, así que podemos decir que, en principio, no tenemos ningún dual inconsistent entre los participantes.

Es cierto que es un tanto difícil querer encasillar a los participantes en este cuadro, sobre todo cuando se carece de justificación a la respuesta, o la justificación es muy vaga, como por ejemplo en "Sí, ¿por qué no?" (Hombre, 32 años). Aquí tomaremos ese tipo de justificación como que no hay nada que impida que una chirigota se haga con tal o cual acento, en lugar de interpretarla más bien como una respuesta pasiva, de alguien quizá no muy interesado en el Carnaval.

Los resultados nos dicen que los encuestados son mayoritariamente thinkers: a la hora de responder, no pesa tanto su relación afectiva entre el Carnaval y el dialecto, sino más bien extrapolan las reglas del Carnaval a otros ámbitos, y eso les parece correcto. Es curioso comentar que en el caso de Madrid hay más feelers que en Almería. Seguramente esto se deba, como mencionamos antes, a la cercanía de los dialectos andaluces, que, aunque son diferentes, en este caso se consideran unidad opuesta a lo que no sea andaluz. De todas maneras, tanto la diferencia entre Almería-Madrid en esta nueva versión, como la de Madrid-Madrid en la original, no son tan significativas como para profundizar en ella. Son detalles que no nos interesan para este estudio.

\section{Relevancia de los Resultados}

Hasta aquí hemos visto que los resultados, tanto los del 2015 como los del 2018, llevan a pensar que la gente de Cádiz es muy abierta con su folklore, pues la mayoría piensa que da igual qué dialecto o acento tenga la chirigota, debería ser igualmente válida. Pero, ¿es esto realmente cierto?

Primeramente, tenemos que pararnos a reflexionar sobre el papel del Carnaval en Cádiz. Es un acontecimiento que tiene lugar todos los años, y que no se limita solamente al mes de febrero, pues las agrupaciones, por ejemplo, se llevan meses ensayando. Las inscripciones al concurso suelen estar entre las 100-150 agrupaciones en los últimos años. Hay decoración en las calles y eventos relacionados con la comida (como la ostioná, la pestiñá, o la erizá). Todos los niños gaditanos crecen sabiendo qué es el Carnaval y, dependiendo de lo mucho o poco que les guste, pueden seguir el concurso por televisión o también salir a la calle disfrazados. El hecho de que esto ocurra sólo en Cádiz forma, 
indudablemente, un vínculo entre acontecimiento y dialecto muy fuerte. ¿Cómo entonces iba a romperse tan fácilmente ese vínculo?

Esta suposición no sólo se apoya en lo que sería lógico, sino que también existen hipótesis en Psicología que lo respaldarían. Tenemos por ejemplo la hipótesis de Trafimow y Sheeran sobre afectividad y cognitividad, donde, en una situación de conflicto, la cognitividad se "traduciría" en afectividad. Esto se ve muy claro en el ejemplo de Josefine (Trafimow y Sheeran, 2005: 65), donde tiene que elegir entre casarse con el hombre que ama, que es pobre, y pasar una vida de necesidades, o casarse con un hombre rico y tener una vida fácil, pero del que no está enamorada. Aquí es cuando Trafimow y Sheeran piensan que, para tomar su decisión, lo que ella hace es ponerle "afecto" a cada una de estas dos opciones, y de ahí decidir cuál es la mejor.

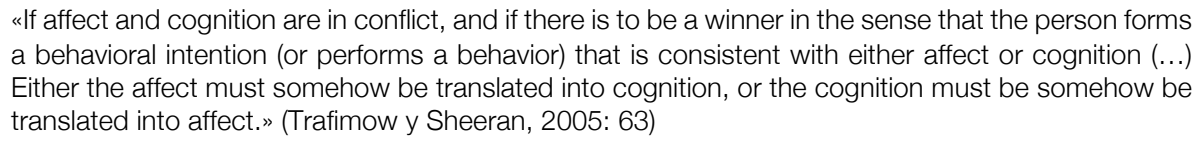

De todo esto deducimos que lo más normal, lo que se esperaría que respondieran, es que el dialecto de Cádiz es el ideal, y que otros no. La pregunta va enfocada a esa parte afectiva, por lo que, en un principio, esperaríamos que se contestaran "afectivamente". Sin embargo, esto no ocurre.

No sólo por lógica e intuición nos resultan raros estos resultados, tenemos también muestras de ello en la vida real. El Concurso de Agrupaciones del Carnaval de Cádiz (a partir de ahora, COAC), es una parte muy importante, casi podríamos decir la esencia y lo que da el pistoletazo de salida al Carnaval en sí. El COAC tiene una tradición de muchos años. La web que vamos a utilizar para contrastar los resultados con la realidad, Carnavaldecadiz.com ${ }^{1}$, tiene una base de datos que se remonta sólo hasta al año 1995. Sin embargo, para nosotros es más que suficiente, y vamos a concentrarnos en el período 2007-2017. Si los resultados de nuestro cuestionario son fieles a la realidad, esperaríamos que:

- Hubiera agrupaciones de distintas provincias en el concurso, no sólo de Cádiz.

- Éstas pudieran, eventualmente, quedar en un puesto similar a las de Cádiz.

La primera suposición la podemos dar por cierta, ya que en esos diez años pasaron por el COAC muchas agrupaciones de distintas provincias: Sevilla, Almería, Cáceres, Ciudad Real, entre otras. Hay incluso agrupaciones que participan varios años seguidos, como son la de Mérida ${ }^{2}$ o la de San José de la Rinconada ${ }^{3}$. Vemos entonces que el Carnaval se presenta como algo abierto a todo aquel que quiera participar, no importa de dónde. Los tipos (disfraces) de estas chirigotas son como las de cualquier otra de Cádiz, y los temas, el humor y la sátira parecen de primeras muy similares, incluso el dialecto que usan.

\footnotetext{
${ }^{1}$ Web COAC http://www.carnavaldecadiz.com

2 http://www.carnavaldecadiz.com/Carnaval2017/Absoluta/Ya no salgo mas.htm

3 http://www.carnavaldecadiz.com/Carnaval2017/Absoluta/No te vayas todavia.htm
} 
Sin embargo, nuestra segunda suposición no se cumple. La mayoría de chirigotas de fuera de la provincia de Cádiz se quedan en preliminares (esto es, la primera fase de la clasificación). Algunas, sobre todo de Sevilla - y esto puede tener que ver, bien con la cercanía geográfica, bien con la cercanía de dialectos, más con la segunda que con la primera, en mi opinión- llegan a pasar a cuartos de final e incluso a semifinales. Sin embargo, a excepción de Los niños cantores de Viena y de Manolete ${ }^{4}$, en el año 2011, no encontramos ninguna chirigota de fuera de Cádiz que pase a la Gran Final. Es cierto que, por número, podríamos pensar que es simplemente cuestión de probabilidad: las chirigotas de Cádiz y provincia representan una enorme mayoría, algunas de ellas muy consolidadas en el mundo del Carnaval, con autores prestigiosos como el Canijo, el Love o el Selu. Pero según muchos de los encuestados, esto no significa que una chirigota de fuera tenga que ser menos buena que una de Cádiz. ¿Por qué no se las ve entonces en puestos altos de la clasificación, incluso en unos cuartos de final?

El caso de nuestro cuestionario no es único. De hecho, se dan muchos casos de cuestionarios en los que las respuestas no reflejan bien lo que ocurre en la realidad (esto es, los resultados y el comportamiento de los individuos no coincide). Constancia de esto tenemos, por lo menos, desde los años 30, con el sociólogo LaPière. Su artículo "Attitudes vs actions" supuso entonces un punto de inflexión en los estudios con encuestas. Su viaje por los EEUU con su pareja de amigos (de nacionalidad china), y el posterior estudio de los cuestionarios enviados a cada uno de los lugares que visitaron, reflejan que el comportamiento se manifiesta de manera muy distinta a como se presenta en este tipo de encuestas. En su caso, que por mucho racismo que pareciera haber en la sociedad americana, en la vida real casi nadie tenía problema para aceptar a una pareja de chinos en su establecimiento.

Para explicar esto, LaPière afirma que, para elaborar las preguntas, hay que hacer "un ajuste verbal a una situación enteramente simbólica" (LaPière 1934: 7). Es justamente lo que hemos hecho en nuestro cuestionario: no les hemos puesto delante a los encuestados una chirigota de Almería y otra de Madrid para preguntarles qué le parecen, sino que hemos recreado una situación, un contexto hipotético donde el encuestado tiene que imaginarse una chirigota con tales características. Unas preguntas simbólicas producirán respuestas simbólicas, y tanto, "no hay necesariamente una correlación entre las palabras y la acción" (LaPière, 1934: 8).

\footnotetext{
"The questionnaire is cheap, easy, and mechanical. The study of human behavior is time consuming, intellectually fatiguing (...). The former method gives quantitative results, the latter mainly qualitative. (...) Yet it would seem far more worthwhile to make a shrewd guess regarding that which is essential than to accurately measure that which is likely to prove quite irrelevant» (LaPière, 1934: 11)
}

Esta es otra cuestión que se plantea a partir de todo esto, que es si el tipo de cuestionario que hacemos va acorde con lo que queremos averiguar. Las preguntas directas, "fáciles y mecánicas", pueden quizás ser buenas a la hora de recopilar datos sobre cualquier otro tema, pero definitivamente pueden arrojar resultados no deseados cuando se trata de

\footnotetext{
${ }^{4}$ http://www.carnavaldecadiz.com/Carnaval2011/Absoluta/Los_ninnos_cantores_de_viena_o_de manolete.php
} 
poner al individuo en "situaciones hipotéticas" - en nuestro caso concreto, de profundizar en la cognitividad y afectividad.

LaPière menciona el término "ajuste verbal": veremos qué papel juega esto más tarde, cuando tratemos sobre distintos tipos de conocimiento en 5.2 .

\section{DISCUSIÓN}

\subsection{Desconocimiento de los distintos dialectos}

Ya hemos mencionado anteriormente que se puede deducir cierto desconocimiento de las características dialectales de Almería, y lo cierto es que probablemente también exista un desconocimiento de cómo es el dialecto de Madrid. Esto lo asumimos partiendo de la base de que, normalmente, uno tiene conciencia de su propio dialecto y de los más cercanos, con los que tiene más contacto, pero no de los que le son más ajenos.

Este desconocimiento podría desde luego considerarse como una explicación a resultados individuales, y por eso es importante mencionarlo y tenerlo en cuenta. No obstante, vemos que este hecho tendería más provocar respuestas afectivas ("el dialecto andaluz es el más adecuado"), y, por tanto, no nos sirve para explicar por qué ese predominio tan grande de las respuestas con base cognitiva.

\subsection{Conocimiento implícito y explícito}

Encontramos una (buena) posible explicación, sin embargo, en la teoría del conocimiento implícito y explícito en Psicología. Según el modelo sociocultural de Rodrigo y Arnay (Portillo González, 2015: 32), en la construcción del conocimiento en la mente se pasa por varios niveles: en el nivel 1 y 2 se aprenden conductas para adaptarse al medio, y esta información se procesa y se almacena para el futuro. En un tercer nivel, la mente formaría "mapas" de la realidad, y estas representaciones son lo que llamamos "conocimiento implícito". En este nivel, las ideas no están disponibles de forma explícita en la conciencia; es decir, no se tiene fácil acceso a ellas y no se espera que sean verbalizadas o justificadas. Eventualmente, en un cuarto nivel, algunas de estas ideas o "mapas" podrían representarse explícitamente, es decir, el individuo sería consciente de ellas y podría deliberarlas y justificarlas.

De todo esto se plantea la siguiente pregunta: ¿qué consideraríamos "conocimiento explícito" en un dialecto, en nuestro caso, el español de España?

- En España hay un español considerado estándar, que consecuentemente es el que tiene más prestigio. Cuanto más se aleje un dialecto de esta versión estándar, menos prestigioso se lo considera.

- El dialecto de Madrid se acerca mucho a la variedad estándar, incluso a veces se asocia con ella. Por lo tanto, se deduce que el dialecto de Madrid tiene mucho prestigio. 
- Los dialectos andaluces se alejan mucho de la variedad estándar, por lo que se consideran mucho menos prestigioso (de hecho, se los considera dialectos estigmatizados).

- Como dialecto estigmatizado, el andaluz lo tenemos asociado a ciertos estereotipos en la televisión y el cine. No se encuentra en programas serios, ni en las noticias a nivel nacional. Si acaso, encontramos su versión "estándar", que no es más que un dialecto andaluz muy suavizado (Ruiz Pareja, 2015: 89).

- Por ello, entre el dialecto de Cádiz y el de Madrid hay grandes diferencias, tanto en prestigio como en el uso.

Todo esto lo podemos considerar como conocimiento explícito, pues lo deducimos de ciertas reglas y podemos razonar por qué las cosas son así. Entonces, ¿qué podemos considerar como "conocimiento implícito"?

- El dialecto de Cádiz "hace gracia", es cómico, es bueno para hacer comedia. Esto puede perfectamente derivarse de esos estereotipos en los medios, que quizás de manera inconsciente nos hacen relacionar lo andaluz con lo "gracioso". Que el gaditano haga gracia no es ningún conocimiento explícito, no nos lo enseñan como tal, no lo aprendemos conscientemente, sino que es más bien un "mapa mental" que la persona se hace desde pequeño, viendo el panorama de dialectos de Cádiz, Andalucía y el resto de España.

- Las chirigotas pretenden hacer gracia, entonces, el de Cádiz es el mejor dialecto para ello.

- Se desarrolla a partir de esto una relación entre "gracia / arte especial" y el propio dialecto. Esta relación se ve reforzada al ser un evento que ocurre cada año y estar muy presente en la vida de los gaditanos.

- Dada la gran diferencia entre Madrid y Cádiz en cuanto al prestigio y al uso, la variedad de madrileña no es adecuada para una chirigota. Quizás la de Almería sí, al compartir (para algunos participantes) ciertos rasgos en común.

Considero, además, el primer punto (del cual se derivan los demás) como conocimiento implícito basándome en Vargas: "They suggest that implicit attitudes influence behaviors that people cannot or do not consciously control (...). Explicit attitudes, on the other hand, influence consciously controlled behaviors.» (Vargas, 2005: 280) Que algo te haga gracia no es algo que se pueda controlar, es automático. A veces incluso ni siquiera la sonrisa o la risa se pueden controlar.

Basándonos en esta división implícito-explícito, podemos analizar algunas de las respuestas que dieron los participantes. Comenzaremos con las negativas:

- Me sorprendo a mí misma, respondiendo a esta pregunta en mi mente, con un "no lo creo". 


\begin{abstract}
Entiendo que las chirigotas, etc. son de Cádiz, pero todavía los/las almerienses podrían hacer un apaño y pasar desapercibidos, ¿pero una chirigota madrileña? Uy. (Mujer, 29 años).

- No creo que funcionara, costaría muchísimo entrar al público, aunque lo importante es el contenido, si el contenido es bueno, da igual el acento (Hombre, 25 años, sobre una chirigota con la variedad de Madrid).

- Creo que no. No me gustaría escuchar "la dije" y cosas así (Mujer, 23 años, suponemos que se refiere al laísmo, un rasgo considerado típico de la variedad de Madrid).

- Sí, aunque creo que perdería algo de gracia, no estaría bien aceptado y el trabajo del autor madrileño deberá ser el triple de bueno para que se tuviese en consideración (Hombre, 28 años. ¿Por qué tendría que ser el trabajo el triple de bueno para tener la misma consideración que el de un autor de Cádiz...?)

- Sí (...) otra cosa es que produzca la misma gracia con otro dialecto o acento a personas de distintas zonas y por consiguiente distintos acentos y dialectos (Mujer, 29 años)
\end{abstract}

Este tipo de respuestas se basan en esa relación afectiva que los hablantes tienen con cada variedad. Podemos afirmar que el tipo de conocimiento que predomina es el implícito, porque el hablante da como razón "no me gustaría", "perdería gracia" y cosas así. El comentario de "me sorprendo a mí misma" es un ejemplo clarísimo de ello, pues la persona en cuestión no es capaz ni siquiera de dar una justificación.

Las respuestas positivas, sin embargo, tienden a ser más "liberales", como podemos ver en El carnaval es universal (Hombre, 29 años) o El cantar no entiende de géneros ni de localidad (Hombre, 28 años) Tenemos varias respuestas del mismo tipo, como Siempre que cumpla los requisitos mínimos: respetuosa, divertida y con ganas de pasarlo bien, puede venir de Tailandia si quiere (Hombre, 27 años) o La manera de hacer chirigota es igual en cualquier parte de España (Mujer, 24 años).

Se deduce, por tanto, que la mayoría de las respuestas positivas tienen que ver con el conocimiento explícito que cada hablante tiene sobre su variedad y las demás. encuestado puede exponer de manera declarativa el porqué de su respuesta, razonar por qué sería o no adecuado, aludiendo a ciertas reglas.

En nuestro caso particular, entonces, las actitudes cognitivas son lo que se considera conocimiento explícito, y las afectivas las que tienen base implícita. Hay evidencias de ello, por ejemplo, en Greenwald (1996), donde habla de las actitudes afectivas como si fueran conocimiento implícito. Doy por hecho que Greenwald se refiere sólo a las actitudes propiamente afectivas, puesto que las compara al mismo nivel con la autoestima y los estereotipos (que son, desde mi punto de vista, claramente afectivos). De igual manera respalda esto Vargas, como vimos anteriormente. No afirmamos rotundamente que las actitudes cognitivas no puedan tener componente implícito, ni tampoco que las afectivas no tengan que ver con el conocimiento explícito: por ejemplo, podríamos pensar que aquellas personas a las que no les gustaría cómo sonaría una chirigota de Madrid, tienen la razón de haber "aprendido" que las chirigotas se hacen con el dialecto de Cádiz. O también, que aquellas a las que no le importaría, quizás hayan crecido en una familia aficionada a chirigotas de fuera de Cádiz (por lo que habrían asimilado inconscientemente que cualquier dialecto es bueno). Pero es cierto que el análisis de los resultados y de sus comentarios sí que da pie a aventurar que gran parte de las actitudes cognitivas y afectivas 
tienen como base, respectivamente, el conocimiento explícito e implícito, al menos en nuestro caso.

La división que hemos hecho aquí no es más que la misma que teníamos en Haddock entre los thinkers y los feelers, pero añadiendo un factor importante a tener en cuenta, que es qué tipo de conocimiento se esconde detrás de estas respuestas. Pero las preguntas que nos planteamos siguen siendo las mismas. ¿Por qué hay mayoría de sí, si en la realidad es que no? ¿Por qué predomina el conocimiento explícito? ¿Qué realidad nos muestran las encuestas? E incluso, ¿podría ser que el formato de la encuesta en sí, fuera lo que arrojara estos resultados?

Si afirmamos, por razones mencionadas anteriormente, que lo que rodea al Carnaval es más afectivo que otra cosa, y las actitudes afectivas son, en este caso concreto, parte del conocimiento implícito, ¿no es contradictorio que estas mismas actitudes puedan ser deliberadas y justificadas? Porque incluso por esto mismo existiría contradicción en la pregunta, al decir pedir que se justifique la respuesta. Si las actitudes afectivas son implícitas, sería bastante difícil "sacarlas al exterior" con una pregunta, que en sí está verbalizada y deliberada para que la respuesta también lo sea. Al pensar en la pregunta, el individuo accede a la información por los niveles 3 y 4 , es decir, puede pensar y a la vez sentir cosas: por ejemplo, puede acordarse de hechos concretos, de aprendizaje en la escuela, sentir molestia o incomodidad por la pregunta en sí... Sin embargo, al contestar, como la información del nivel 3 no se puede expresar ni justificar de forma declarativa, el individuo tiene que acceder por el nivel 4, lo que hace que estos valores afectivos y cognitivos choquen y den lugar a respuestas, supuestamente, erróneas (comparadas con la realidad).

\begin{abstract}
Me sorprendo a mi misma, respondiendo a esta pregunta en mi mente, con un "no lo creo". Entiendo que las chirigotas, etc. son de Cádiz, pero todavía los/as almerienses podrían hacer un apaño y pasar desapercibidos, ¿pero una chirigota madrileña?... Uy.Me sorprendo a mi misma, respondiendo a esta pregunta en mi mente, con un "no lo creo". Entiendo que las chirigotas, etc. son de Cádiz, pero todavía los/as almerienses podrían hacer un apaño y pasar desapercibidos, ¿pero una chirigota madrileña?... Uy.Me sorprendo a mi misma, respondiendo a esta pregunta en mi mente, con un "no lo creo". Entiendo que las chirigotas, etc. son de Cádiz, pero todavía los/as almerienses podrían hacer un apaño y pasar desapercibidos, ¿pero una chirigota madrileña?...
\end{abstract}

\title{
5.3 Greenwald y la "atención"
}

Por último, encontramos en Greenwald diferentes hipótesis que refuerzan lo que hemos visto hasta ahora, en concreto las que se basan en el hecho de que "prestar atención a la fuente del efecto implícito reduce dicho efecto", tal y como él lo define. "When attention is focused on the source of an implicit effect that interferes with judgment, that interference is reduced or eliminated (and sometimes even reversed).» (Greenwald, 1996: 28). En nuestro caso, lo que se ha hecho con esas preguntas es centrar la atención en el componente afectivo del Carnaval (esto es, conocimiento implícito), por lo que es muy probable que esa afectividad se reduzca o elimine. En las palabras de Greenwald, en muchas de las respuestas no sólo se ha eliminado, sino que la afectividad se ha invertido, pasando a ser cognitividad. 
Algo que podríamos pensar es que, si hubiéramos omitido la aclaración "justifica tu respuesta", quizás habríamos obtenido más resultados feelers que thinkers. El hecho de tener que justificar la respuesta exige más atención por parte del hablante en los rasgos implícitos de la actitud afectiva. Esto no es del todo descartable, aunque sí es cierto que en el cuestionario original se obtuvieron resultados similares, sin incluir esta aclaración.

La razón de que la atención atenúe o elimine la afectividad puede hallarse en lo mencionado en 5.2, sobre el acceso a la información en los distintos niveles, y no hace sino añadir más argumentos a favor de la hipótesis que hemos estado barajando. Cuanto más énfasis se haga a lo afectivo en la pregunta, más intentará el hablante responderla y justificarla; sin embargo, la afectividad y la cognitividad están en distintos niveles, no se puede acceder a ellas de la misma forma, y, por tanto, los resultados no son del todo fiables a la hora de anticipar un comportamiento.

Esta hipótesis estaría complementada con lo que tanto Greenwald como Trafimow llaman recall (Trafimow y Sheeran, 2005: 60): las actitudes evocan actitudes del mismo tipo. Esto a primera vista parece ser justo lo contrario, pues lo que aquí se aprecia es que las supuestas actitudes afectivas evocan respuestas cognitivas. Lo que se deduce, entonces, es que, en este tipo concreto de preguntas directas, sí que existiría ese recall, y sí que evocaría afectividad, pero lo que esto implica es ponerle más atención al aspecto implícito de la actitud. Por lo tanto, la afectividad se nos invierte y se transforma en respuestas de naturaleza cognitiva.

\subsection{Otro punto de vista: Schuman, actitudes vs. actitudes}

El trabajo de Schuman "Attitudes vs. actions versus attitudes vs. attitudes" (1972) es un comentario al estudio de LaPière, y nos da otro punto de vista sobre cómo este tipo de cuestionarios puede reflejar la realidad, aunque los resultados sean a primera vista "contrarios" a ella.

En su trabajo presenta tres preguntas de un cuestionario realizado en los años sesenta en Detroit, sobre las actitudes raciales. Las respuestas a estas preguntas dan la impresión de ser muy contradictorias entre sí. ¿Cómo puede ser que la mayoría de los encuestados vea mal que un jefe juzgue a un potencial empleado por su color de piel, y que, sin embargo, estos mismos cambien de opinión cuando esta decisión está "justificada"? (Schuman, 1972: 348-349). Aquí claramente lo que existe es un choque de valores, entre el racismo y el bien común. Esto es precisamente lo que se pretendía en nuestro cuestionario: que el hablante pusiera, a un lado de la balanza, su relación afectiva con el dialecto, y en el otro, el conocimiento que tiene de su lengua y la realidad.

Lo que él afirma es, básicamente, que en estas preguntas es fácil simular algunas presiones de la vida diaria y, al contrario de lo que afirmaba LaPière, no importa que esta situación sea hipotética. La aparente inconsistencia que se puede apreciar entre respuestas del mismo cuestionario, o entre los resultados y la realidad, es el resultado del esfuerzo del 
individuo para reconciliar dos o más valores en conflicto. Lo que se nos presenta es la visión del individuo sobre el conflicto en sí, no el comportamiento que se tendría en una situación real.

"Our examples do suggest that an attitude survey can represent these conflicts with a good deal of success, provided only that we are willing to build into our questionnaires the problems and not merely the platitudes that life holds for most individuals» (Schuman, 1972: 254). Es decir, aunque los resultados parezcan no coincidir con la realidad, siempre están acordes con ella de alguna forma. De aquí podemos darles otro enfoque a los resultados de nuestra encuesta: puede que sí sea verdad que los gaditanos sean muy abiertos a que otros participen en el Carnaval; puede que sí sea cierto que no tengan ningún problema a la hora de que una chirigota "de fuera" venga a cantar a Cádiz. Otro asunto sería que les resultara agradable, les hiciera gracia o lo que sea. El cuestionario mostraría otra cara de la realidad, mostraría cómo manejan los gaditanos este tipo de conflicto interno.

Este tipo de cuestionario "mecánico" e "hipotético", según LaPière, mostraría la realidad desde el punto de vista del conflicto, no del comportamiento. Si ese no es nuestro objetivo, este tipo de cuestionarios no nos sirve. Sin embargo, el análisis de Ruiz Pareja (2015) no tenía un objetivo concreto, simplemente era un análisis abierto, en el que las conclusiones a estas preguntas podrían haberse abarcado libremente de una manera o de otra.

\section{CONCLUSIONES}

La finalidad del cuestionario era ver qué actitudes predominan en los hablantes a la hora de enfrentarse a una rotura en la relación dialecto-elemento del folklore, e intentar encontrarle una explicación. Parecía que las actitudes cognitivas predominaran sobre las afectivas; sin embargo, al analizarlo en profundidad y compararlo con la realidad, esto no hace mucho sentido. Tanto los hechos (los resultados del COAC) como una aproximación teórica sobre la teoría de la cognitividad y la afectividad (Trafimow y Sheeran), nos indican que hay algo "extraño" en los resultados. ¿Cuál es la razón?

Una posible explicación es la poca veracidad que tiene una actitud afectiva cuando se verbaliza. Verbalizamos la situación hipotética (la pregunta), donde entran en juego dos valores opuestos. El individuo piensa sobre la pregunta, y encuentra un conflicto. Esto, en teoría, se suele resolver traduciéndolo en afectividad, según Trafimow. Sin embargo, las actitudes afectivas no pueden verbalizarse, justificarse o expresarse de manera declarativa, pues hemos visto que pertenecen al ámbito del conocimiento implícito. La atención que el individuo le pone y la reflexión que ello conlleva (Greenwald) hacen que el efecto "afectivo" se reduzca o desaparezca. Ergo, lo que queda es la cognitividad. Y cognitivamente, no hay nada que diga que las chirigotas no pueden ser de tal o cual sitio, o con tal o cual dialecto. Sólo algo "cognitivo" puede pasar el filtro del conocimiento explícito; algo afectivo, no. Ésta pudiera ser la razón de que obtengamos resultados no acordes con la realidad en este tipo de cuestionarios, es decir, no nos sirven para anticipar comportamientos. 
Sin embargo, tenemos también el aporte de Schuman, del que podríamos concluir que este tipo de cuestionarios no arroja resultados absurdos del todo: habría que ahondar un poco más en la situación de los hablantes de Cádiz para obtener conclusiones, pero desde luego, saber que existe este conflicto de valores, esas "actitudes vs. actitudes" de las que habla Schuman, y analizar cómo el hablante se inclina por una o por otra, nos ofrece otro punto de vista sobre la realidad que merece la pena tener en cuenta. Los resultados son siempre acordes con la realidad de una u otra manera.

Otra conclusión que podemos sacar de este estudio es que, según qué resultados se busquen, se deberían usar distintos tipos de cuestionario. Un cuestionario del tipo "sí/no y justifique su respuesta", es poco probable que vaya a anticipar comportamientos en la vida real en situaciones donde haya un conflicto afectivo-cognitivo. En estos casos, seguramente sea mejor hacer preguntas no tan directas, quizás más prácticas, como crear una situación hipotética mucho más detallada y con preguntas que vayan más allá del sí y el no, o donde incluso se vea el comportamiento del hablante directamente (enseñarle una chirigota de fuera de Cádiz, sin que se sepa, y preguntar qué le parece, por ejemplo). Sin embargo, el tipo de cuestionario que nosotros tenemos es adecuado si queremos ver de qué manera los hablantes de cierta variedad abordan un conflicto de valores, y las conclusiones que queramos sacar a partir de aquí.

\section{BIBLIOGRAFÍA}

Coloma, Germán (2013): «Los continuos dialectales de la lengua española», De Gruyter: Zeitschrift für romanische Philologie, 129, 2, 366-382.

Greenwald, Anthony y Mahzarin R. Banaji (1995): «Implicit social cognition: attitudes, self-esteem and stereotypes», Psychological Review, 102 (1), 4-27.

Haddock, Geoffrey y Huskinson, Thomas L.H. (2005): «Individual differences in attitude structure», en Haddock, Geoffrey y Gregory R. Maio, eds., Contemporary perspectives on the Psychology of Attitudes, New York, Psychology Press, 35-56.

LaPière, Richard T. (1934): «Attitudes versus actions", International Journal of Epidemiology, 39, 1, 1 February 2010, 7-11, https://doi.org/10.1093/ije/dyp398.

Lord, Charles G. (2005): "The role of exemplar stability in attitude consistency and attitude change», en Haddock, Geoffrey y Gregory R. Maio, eds., Contemporary perspectives on the Psychology of Attitudes, New York, Psychology Press, 299-323.

Portillo González, Adriano Manuel (2015): «La construcción de la mente humana: del conocimiento implícito al conocimiento explícito", Realitas, Revista de Ciencias Sociales, Humanas y Artes, 3 (1), 31-16.

Rodrigo, María José y Arnay, José (1997): La construcción del conocimiento escolar, Barcelona, Paidós.

Ruiz Pareja, Raquel (2015): Estudio sobre las actitudes lingüísticas en Andalucía: andaluz oriental y andaluz occidental, Trabajo final de máster, The Artic University of Norway, [en línea]:

https://munin.uit.no/bitstream/handle/10037/9 194/thesis.pdf?sequence $=1$

Schuman, Howard (1972): «Attitudes vs. Actions versus Attitudes vs. Attitudes», Public Opinion Quarterly, 36, 3, 347-354.

Trafimow, David y Paschal Sheeran (2005): «A theory about the translation of cognition into affect and behavior», en Haddock, Geoffrey y Gregory R. Maio, eds., Contemporary perspectives on the Psychology of Attitudes, New York, Psychology Press, 57-75

Vargas, P.T. (2005): «The relationship between implicit attitudes and behavior. Some lessons from the past, and directions for the future", en Haddock, Geoffrey y Gregory R. Maio, eds., Contemporary perspectives on the Psychology 
Resultados vs realidad: un ejemplo en las actitudes lingüísticas | R. Ruiz Pareja

of Attitudes, New York, Psychology Press, 275297.
Web del Carnaval de http://www.carnavaldecadiz.com/
Cádiz: 